\title{
Clinical implementation of a novel applicator in high-dose-rate brachytherapy treatment of esophageal cancer
}

\author{
Ivan M. Buzurovic, PhD, Jorgen L. Hansen, MS, Mandar S. Bhagwat, PhD, Desmond A. O'Farrell, MS, \\ Scott Friesen, MS, Thomas C. Harris, MS, Antonio L. Damato, PhD, Robert A. Cormack, PhD, Neil E. Martin, MD, MPH, \\ Phillip M. Devlin, MD \\ Department of Radiation Oncology, Dana Farber/Brigham and Women's Cancer Center, Harvard Medical School, Boston, MA, USA
}

\begin{abstract}
Purpose: In this study, we present the clinical implementation of a novel transoral balloon centering esophageal applicator (BCEA) and the initial clinical experience in high-dose-rate (HDR) brachytherapy treatment of esophageal cancer, using this applicator.

Material and methods: Acceptance testing and commissioning of the BCEA were performed prior to clinical use. Full performance testing was conducted including measurements of the dimensions and the catheter diameter, evaluation of the inflatable balloon consistency, visibility of the radio-opaque markers, congruence of the markers, absolute and relative accuracy of the HDR source in the applicator using the radiochromic film and source position simulator, visibility and digitization of the applicator on the computed tomography (CT) images under the clinical conditions, and reproducibility of the offset. Clinical placement of the applicator, treatment planning, treatment delivery, and patient's response to the treatment were elaborated as well.

Results: The experiments showed sub-millimeter accuracy in the source positioning with distal position at $1270 \mathrm{~mm}$. The digitization (catheter reconstruction) was uncomplicated due to the good visibility of markers. The treatment planning resulted in a favorable dose distribution. This finding was pronounced for the treatment of the curvy anatomy of the lesion due to the improved repeatability and consistency of the delivered fractional dose to the patient, since the radioactive source was placed centrally within the lumen with respect to the clinical target due to the five inflatable balloons.

Conclusions: The consistency of the BCEA positioning resulted in the possibility to deliver optimized non-uniform dose along the catheter, which resulted in an increase of the dose to the cancerous tissue and lower doses to healthy tissue. A larger number of patients and long-term follow-up will be required to investigate if the delivered optimized treatment can lead to improved clinical outcomes.
\end{abstract}

J Contemp Brachytherapy 2016; 8, 4: 319-325 DOI: 10.5114/jcb.2016.61933

Key words: brachytherapy, esophageal cancer, HDR, optimization.

\section{Purpose}

Esophageal cancer develops in the mucosa of the esophagus and spreads towards the muscle layer. The nonsurgical treatment for localized, deeply invasive esophageal cancer has been external beam radiation therapy (EBRT) and concurrent chemotherapy [1]. Recently, intraluminal brachytherapy showed a strong potential for the improvement of the therapeutic ratio [2]. It was found that the fractionated high-dose-rate (HDR) brachytherapy offered beneficial palliation for a longer period of time with more durable symptom control [3,4]. A similar finding was found for advanced unresectable esophageal cancer in previously irradiated patients $[5,6]$. Hihg-dose-rate brachytherapy may be a useful salvage treatment option for inoperable patients diagnosed with local esophageal cancer [6]. Although better local control can be achieved with higher brachytherapy dose, this increases the risk of acute morbidity [4,7] and late morbidity, especially in the setting of recurrence cancer [4]. It was found that the moderate dose of EBRT and HDR brachytherapy could give a better local response than EBRT alone [8]. The results in a large cohort of patients indicated that HDR brachytherapy alone was an effective method for the palliation of advanced esophageal cancer [9]. Similar long-term results were reported in favor of treatments involving concurrent chemoradiotherapy followed by 
HDR brachytherapy [10]. Although brachytherapy was found to be preferable, there are studies (such as [11,12]) suggesting that stent placement may play an important role for the palliation of disease. In that case, the prognostic models were used as evidence-based tools in decision making. However, the health-related life quality was reported to be improved in patients treated with the HDR brachytherapy. Recent studies suggested the usage of ${ }^{252} \mathrm{Cf}$ neutron brachytherapy combined with EBRT for esophageal cancer. The treatment resulted in favorable local control and long-term survival rates with tolerable side effects [13].

Patient selection, timing of brachytherapy, and dose specifications were well documented $[1,2,14]$. Clinicians continue to urge caution in using brachytherapy treatment techniques since severe toxicity can occur post treatment $[10,14,15]$. Therefore, the addition of brachytherapy, with consequently high surface doses, should be limited to well-selected patients [15].

For that reason, the clinical implementation and accuracy in dose delivery is crucial for favorable treatment outcomes. The radiation dose is delivered using esophageal transoral or transnasal applicators with an external diameter of $0.6-1 \mathrm{~cm}$. Ideally, the single channel applicator needs to be placed centrally in the lumen of the esophagus; however, there exists a possibility that the applicator will be closer to one side of the lumen, delivering a larger dose to the epithelium, lamina propria, and muscularis mucosa, resulting in local esophageal complications. In those cases, stricture formation, fistula, and esophageal ulceration are the common late toxicities of HDR brachytherapy [16]. A possible difference in the delivered dose is caused by disagreements in the choice of the dose point (i.e. mucosal surface or certain distance from the central line of the applicator) in various institutions, as reported in [17]. For instance, it was reported in the long-term experience with esophageal brachytherapy treatment [18] that radiation was delivered at a level of $5 \mathrm{~mm}$ below the surface of the mucosa. However, no correlation was found between the post-treatment complications and the diameter of the brachytherapy applicator [19]. In most of the HDR brachytherapy treatments, 3D treatment plans were generated using computed tomography (CT) images; however, magnetic resonance imaging (MRI) can be used to assist the localization of the tumor and the applicator [20]. The treatment planning for the esophageal cancer patient is performed using the TG-43 formalism [21], since the dose calculation accuracy of the TPS was confirmed in a homogeneous medium [22]. Overall, this HDR treatment is demonstrated to be well-tolerated and effective for superficial primary and recurrent esophageal cancer in inoperable patients $[23,24]$. The authors concluded that dose escalation with larger diameter applicators may allow for improved therapeutic coverage without exceeding the organs at risk tolerances [23]. The latest research in the combined approach (EBRT and HDR) to palliation in esophageal cancer together with the review of the current techniques is reported in $[25,26]$.

The purpose of the study is to report the initial experience in the treatment of the locally advanced esopha- geal carcinoma with HDR brachytherapy, using a novel transoral balloon centering esophageal applicator (BCEA; E-APPтM, Ancer Medical, USA). In this study, we report the commissioning procedure, treatment planning, HDR brachytherapy treatment delivery, and initial patient response using the novel BCEA. The experience in the treatment of esophageal cancer using a standard intraluminal esophageal applicator (EA) was summarized in [27]. The comparison between the standard EA and the BCEA is elaborated in all phases of the clinical workflow including the treatment of the patient diagnosed with esophageal cancer.

\section{Material and methods}

\section{Patient}

Initially, the patient was diagnosed with an adenocarcinoma of the distal esophagus. The pathological TNM classification was T3N2MX. The patient underwent chemoradiotherapy (50 Gy) preoperatively for locally advanced esophageal carcinoma, followed by a partial esophagectomy. Two months after surgery, the patient was diagnosed with a recurrent neoplasm of the middle third of the esophagus, as recurrent adenocarcinoma at the anastomosis. Computed tomography/positron emission tomography (CT/PET) imaging showed an avid lesion at the anastomosis. A combined external beam and brachytherapy approach was created as salvage therapy. The patient first received 26 Gy salvage external beam radiation therapy to the maximal normal tissue dose constraint. At the endoscopic reevaluation after therapy, there was persistent tumor of $5.5 \mathrm{~cm}$ in greatest dimension (gross tumor volume). High-dose-rate brachytherapy was used to bring the total equivalent dose up 50 Gy for the recurrence. The clinical target length was $9 \mathrm{~cm}$, so the total treatment length (planning target volume) includes a $3 \mathrm{~cm}$ margin both proximally and distally, which resulted in a total of $15 \mathrm{~cm}$ treatment length.

\section{Acceptance and commissioning of the balloon centering esophageal applicator}

To decrease the dose to the organs at risk in the upper gastrointestinal region, we used the novel disposable brachytherapy BCEA with five independently inflatable balloons (Figure 1) to allow for the central placement of the radioactive source during treatment. The applicator was designed for treatment lengths greater than $10 \mathrm{~cm}$. The BCEA allows for the treatments outside the balloon region with the constraint where the BCEA becomes similar to the standard EA. Therefore, the dose optimization outside the balloon region should be avoided due to the complicated position reproducibility. A diameter of the BCEA is $0.5 \mathrm{~cm}$. The visibility of the catheter on the CT images is obtained with $12 \mathrm{CT}$ and MRI compatible radio- opaque markers. The first marker is placed at the tip of the BCEA, the next one is $0.3 \mathrm{~cm}$ proximal, and each subsequent marker is $1 \mathrm{~cm}$ apart (Figure 1). The applicator has inflatable ports at the proximal end for filling the balloons with water before the treatment delivery. 
A

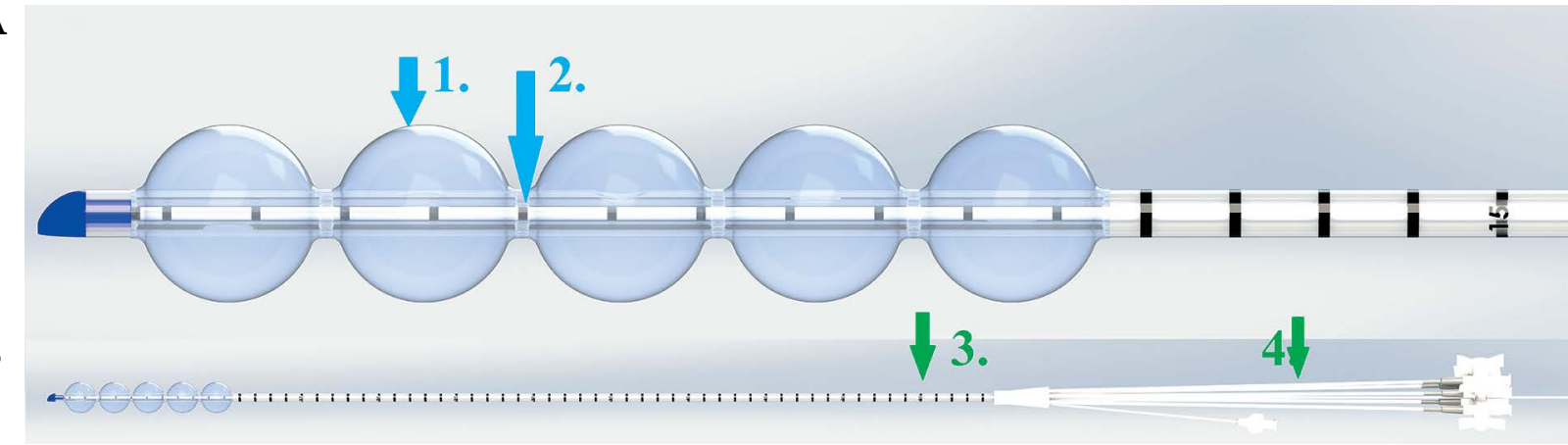

Fig. 1. Transoral balloon centering esophageal applicator. A) Five inflatable balloons (1) allow for reproducibility of the treatment setup. Radio-opaque contrast markers are visible on computed tomography and magnetic resonance images (2); B) Full view - a catheter (3) and the inflatable ports (4). Image supplied by Ancer Medical (Hialeah, FL, USA)

The BCEA comes in a sterile package, so one applicator was used for acceptance testing and commissioning. During the acceptance testing, the performance evaluation and familiarization with the proper function of the BCEA was conducted. The following was evaluated: the dimensions, catheter diameter, balloon consistency in inflated and non-inflated conditions, diameter of the balloon after inflation, marker placement stability, and the absolute and relative positioning of the markers (marker congruence). Prior to treatment, the applicator was commissioned and tested for clinical implementations. The following was tested: a) visibility of the radio-opaque markers in the CT images to assure the proper placement; b) repeatability, consistency, and CT visibility of the water inflatable balloons; c) absolute and relative accuracy (sequencing) of the source positioning in the applicator using the source position simulator and radiochromic film (EBT3 GafChromic $^{\mathrm{TM}}$, Ashland, Covington, USA). In addition, the usability and accuracy of 12 radio-opaque markers on the exterior side of the catheters were tested for proper source placement in the clinical target. The additional goal of this test was to determine the reproducibility of the offset. Due to the possibility of the subjective judgment for some tests, the data were analyzed by three independent observers. The test was marked as 'pass' only if an interobserver agreement was established.

\section{Applicator placement}

The BCEA design includes two lumens: a central lumen to contain the HDR radioactive source, and the second lumen through which the guide wire passes. Unlike with the transnasal insertion where the endoscope would be placed via the anesthetized nose past turbinates and nasopharynx behind the larynx and into the esophagus, this applicator was placed transorally. This avoided the customary trauma, bleeding, and pain. The scope was placed transorally and the guide wire passed through the side port. The scope was removed and the applicator was placed over the guide wire. The scope was then reinserted via the mouth alongside the applicator to direct final placement. At the end of the procedure, the guide wire and the scope were removed.

\section{Treatment planning}

For the treatment planning, we used Oncentra Brachy planning system, Version 4.3.0.410 (Nucletron, an Elekta company, Elekta AB, Stockholm, Sweden). 3D treatment plan was generated using a CT image set with a $1.25 \mathrm{~mm}$ slice thickness to allow for the accurate digitization of the catheter. The prescription dose for the HDR treatments was $15 \mathrm{~Gy}$ in 3 fractions to the distal esophagus with a $5 \mathrm{~cm}$ offset from the end of the applicator. The treatment frequency was twice daily, at least 6 hours apart. The treatment (target) length was $15 \mathrm{~cm}$, which resulted in 31 dwell positions having a step size of $0.5 \mathrm{~cm}$. The treatment length was defined as a pretreatment tumor length with 1-2 cm distal, and proximal margin determined by pretreatment imaging and confirmed by the CT images. With the standard EA, the dose point is defined at the mucosal surface or a certain distance from the central line of the applicator with identical dwell times along the treatment length. This was mostly done to minimize the uncertainties in dose delivery related to the positioning of the EA inside the esophagus. The BCEA positions the catheter centrally when the balloons are inflated. Due to that fact, the treatment plan can be additionally optimized for improved dose distribution and conformality. The prescription dose was planned to be delivered to the diameter of $1 \mathrm{~cm}$ with respect to the central catheter with an additional optimization to avoid the critical anatomical structures such as the heart, lung, pharyngeal constrictor, and spine. The dose calculation was performed using the TG-43 formalism that includes the anisotropy corrections. Heterogeneity corrections were not included in the brachytherapy dose calculation.

\section{Results}

\section{Acceptance and commissioning of the balloon centering esophageal applicator}

Acceptance testing confirmed the BCEA specification: the catheter diameter was $0.5 \mathrm{~cm}$. The diameter of the inflated balloons was $2 \mathrm{~cm}$ when $5 \mathrm{~cm}^{3}$ of sterile water was injected through the baffle inflation ports, filling the inflation catheter, and the balloon itself. All radio-opaque markers were well visible on the scout images and on the 

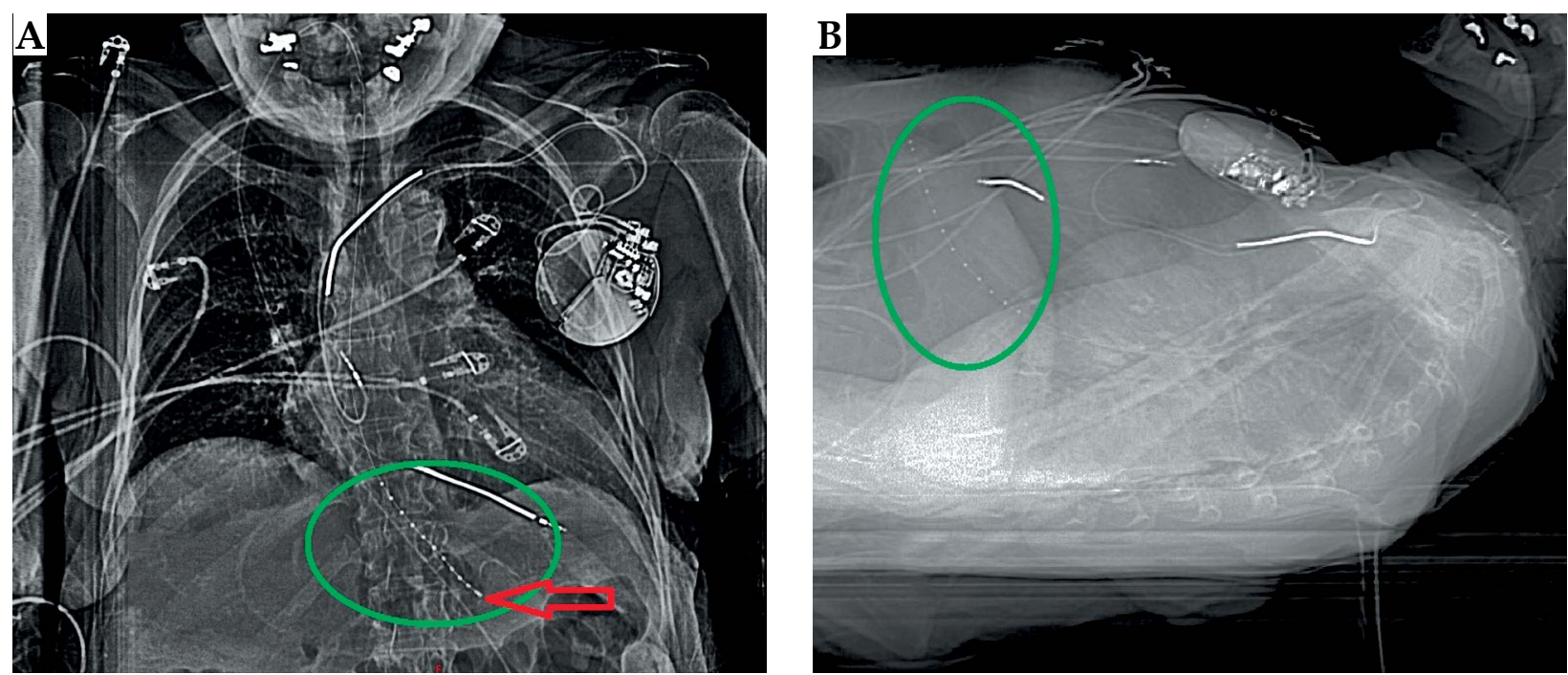

Fig. 2. Anterior-posterior and lateral scout taken prior to treatment. The green markers and red arrow point to the radio-opaque markers. The markers are visible under various window and level setup as shown in A) and B) sections

CT in laboratory conditions. The relative positioning of the markers was within specification: the distance of the first and second markers was $0.3 \pm 0.02 \mathrm{~cm}$, and the subsequent markers were $1 \pm 0.03 \mathrm{~cm}$ apart. Multiple independent measurements were taken using a ruler and CT images by three physicists.

The commissioning revealed good positioning accuracy and visibility of the markers in the CT images. The visibility of the markers in the clinical setup is presented in Figure 2. The scout images are taken prior to the HDR brachytherapy treatment. The radio-opaque markers of the applicators were visible in various window and level setup configurations of the CT images. The anterior-posterior and lateral scout images are presented in Figures $2 \mathrm{~A}$ and $2 \mathrm{~B}$, respectively. The imaging of the applicator and measurements of the relative distance on the CT images were confirmed, and the results were at the same

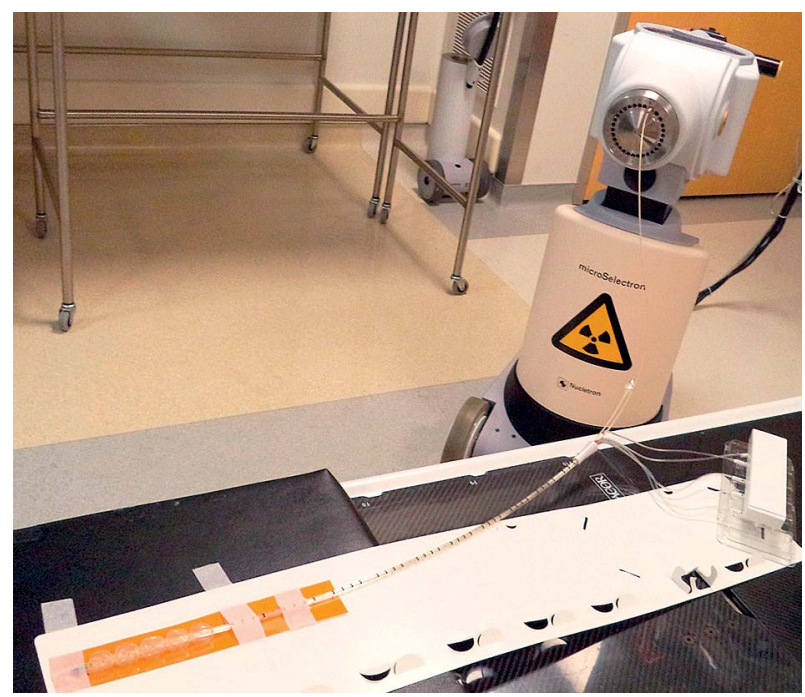

Fig. 3. The experimental setup for testing the absolute and relative accuracy of the radioactive source placement. The treatment offset was tested using this experimental setup level as on the physical measurements. Since the manufacturers recommendation was to inflate the balloons before treatment and deflate them after each treatment fraction, we confirmed the consistency of the applicator (balloons) after several inflations and deflations of the balloons. The absolute positioning of the source at the most distal position was crucial to establish the maximum treatment length distance and to define possible offsets in treatment delivery. For that purpose, radiochromic film was used. The experimental setup was presented in Figure 3. The source was sent to various distances initially determined using the source position simulator. The purpose of this test was to determine the exact position of the source at its distal end. The test results were presented in Figure 4. It was revealed that the distal position of the source ${ }^{192} \mathrm{Ir}$ (source extension) was $1270 \mathrm{~mm}$ for the microSelectron V.2. afterloader (Nucletron, an Elekta company, Elekta AB, Stockholm, Sweden) using a film and source position simulator. When the BCEA was attached to the afterloader and a $1270 \mathrm{~mm}$ source extension was used, the additional test results showed sub-millimeter accuracy in the source positioning of the whole system.

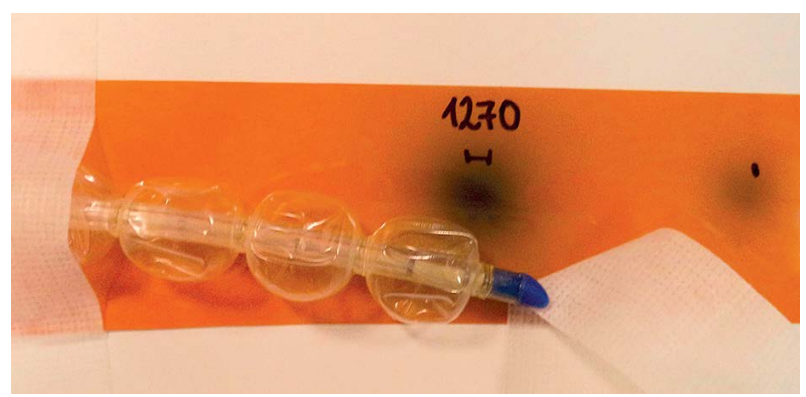

Fig. 4. The distal position of the source - source extension. The value obtained in this test is entered into the treatment planning system to avoid the geometric displacement of the source during the treatment 


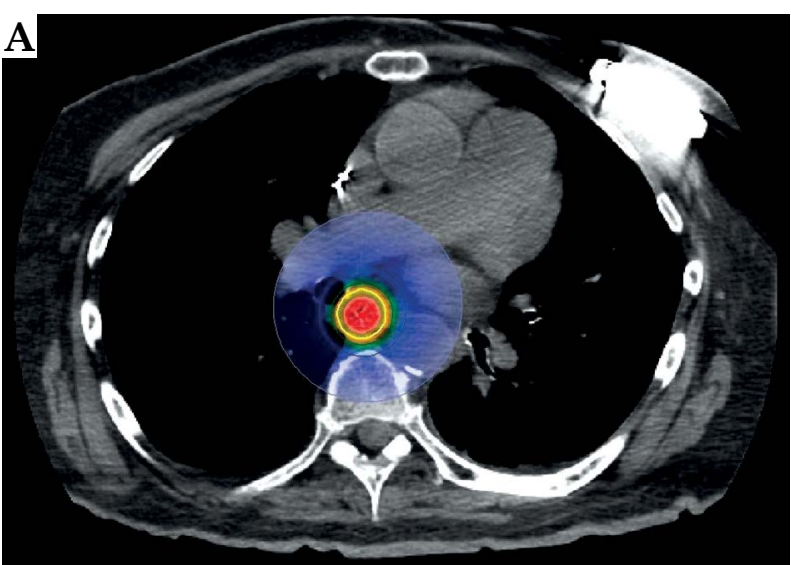

Fig. 5. A) Axial and B) sagittal images show the optimized dose distribution. The centrally placed catheter inside the esophagus lumen resulted in enhanced dose distribution and reproducibility in multi fractional treatment

\section{Treatment planning}

The digitization (catheter reconstruction) was uncomplicated due to good visibility of the markers, and the treatment planning resulted in a favorable dose distribution (Figure 5). Figure 5 presents the axial and sagittal slices of the treatment plan. Based on the CT images, it was confirmed that the BCEA was placed centrally due to the inflatable balloons. That allowed us additional optimization in that region, since the source was positioned centrally with respect to the lumen during the treatment (Figure 6). Unlike in the standard practice where the dwell times for the source were identical due to the local uncertainties of the catheter placement from one fraction to the other, the treatment planning resulted in different dwell times in the regions of the balloons, allowing for an additional adjustment of the dose. For instance, in the balloon region (distal target) $100 \%$ of the prescription dose was at a distance of $1 \mathrm{~cm}$ to $1.8 \mathrm{~cm}$ from the source; however, this distance was $1 \mathrm{~cm}$ outside the balloon region (proximal target). This indicates that the clinical target volume would receive a lower dose if the patient was treated with the standard EA. Therefore,

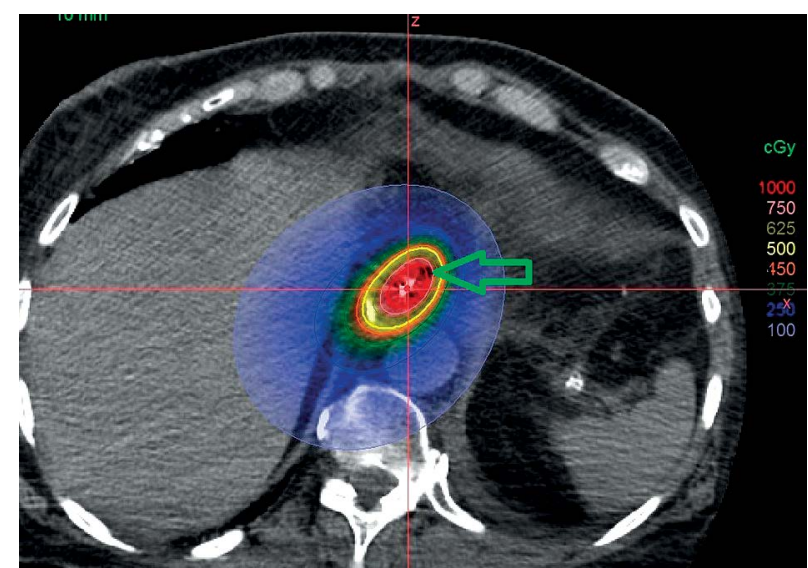

Fig. 6. Axial computed tomography image showing the central placement of the radiation source (red dwell) and with respect to the inflated balloon (green arrow)

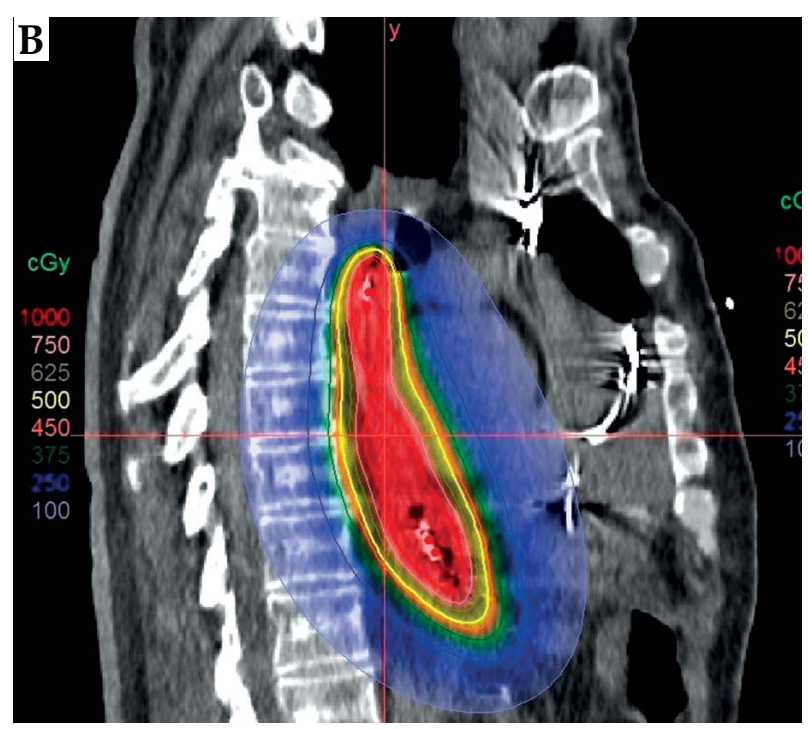

the plan optimization resulted in an enhanced optimized dose distribution in comparison to the case when a standard esophageal applicator was used. The dosimetry of the organs at risk (cord and lungs) is presented in Table 1.

\section{Treatment delivery}

Unlike standard endobronchial and esophagus brachytherapy treatment in which the applicators are placed transnasally, this BCEA was placed transorally, allowing for less irritation of the nose, nasopharynx, and oropharynx. The applicator stayed in place during the whole multi-fractional course of treatment. The BCEA is designed to be a self-anchoring applicator; however, the position of the applicator was verified prior to each fraction, as elaborated in the sequel. Each balloon was filled with $5 \mathrm{~cm}^{3}$ of water prior to the delivery of each fraction (Figure 7 ) to place the catheter centrally while the distance from healthy tissue was maintained; the balloons were emptied after each fraction. Prior to the delivery of each fraction, the catheter measurements were taken for the quality assurance purpose.

Table 1. The dosimetry of the organs at risk ROI Dose [\%] Dose [cGy] Volume [\%] Volume [cm $\left.{ }^{3}\right]$

\begin{tabular}{lcccc}
\hline Cord & 19.54 & 97.70 & 5.00 & 1.87 \\
\hline Cord & 19.43 & 97.15 & 5.34 & 2.00 \\
\hline Cord & 23.87 & 119.37 & 0.27 & 0.10 \\
\hline Cord & 21.17 & 105.83 & 2.00 & 0.75 \\
\hline Cord & 20.66 & 103.28 & 2.67 & 1.00 \\
\hline Lung & 5.98 & 29.91 & 50.00 & 1308.15 \\
\hline Lung & 8.46 & 42.29 & 30.00 & 784.89 \\
\hline Lung & 10.52 & 52.61 & 20.00 & 523.26 \\
\hline Lung & 13.00 & 65.01 & 13.00 & 340.12 \\
\hline Lung & 19.78 & 98.92 & 5.00 & 130.81
\end{tabular}




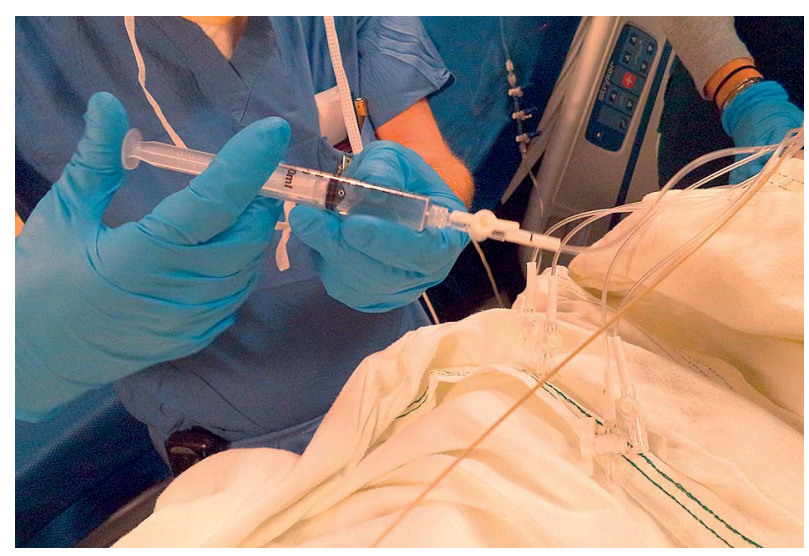

Fig. 7. Balloons were filled with water prior to delivery of each treatment fraction

The position of the applicator and the balloon diameters was verified before the treatments using the planning CT images and the CT images obtained prior to each fraction. Three methods were used to verify the proper positioning of the BCEA prior to each treatment: a) using an external marker to verify the length of the catheter in the patient; b) evaluation of the position of 12 radio-opaque markers on the exterior side of the catheter in the CT images, and c) measurements of the diameters of the balloons on CT images after inflation to confirm that they were properly inflated. The total treatment time for each fraction was less than 6 minutes with a source activity of $9.2 \mathrm{Ci}$. The patient tolerated the treatment well in the supine position, and did not experience dysphagia or increased discomfort during the treatment.

\section{Discussion}

The novel design of BCEA requires some changes in the clinical workflow including the applicator placement, treatment planning, and treatment delivery. The BCEA is placed transorally. Although this technique is not novel in esophageal cancer treatments [1], this allows for maintaining the applicator in place during the whole course of treatment, only the balloons were deflated (the sterile water was removed). The benefit of this approach is the consistency of the intra-fractional positioning and the accuracy of the delivery of the intended radiation dose.

Classically, one of the limitations for the deployment of esophageal brachytherapy has been the difficulties associated with the placement and tolerance of the transnasal applicator [27]. The common adverse effects included significant pain on placement and for the duration of its indwelling. Nasal bleeding, often significant, can be seen from both the scope and catheter placement. There is a frequent need of significant pain medicine to tolerate this procedure.

These effects are pronounced if the applicator is kept in place for an extended period of time. In the presented case, the applicator stayed in place during the whole multi-fractional course of treatment, which minimizes the possibility for such occurrences. In comparison to the transnasal approach, the advancement of the transoral applicator takes so much discomfort and risk away. In this setting, the nose, turbinates, and nasopharynx are never traversed.
This applicator can potentially cause issues if the esophageal mucosa gets compressed between the inflatable balloons. However, we did not experience any of these complications, since the mucosa gets evenly redistributed around the applicator during the treatment. Upon deflation of the balloons, the tissue gets released immediately after delivery of each fraction.

An additional step in treatment plan generation for esophageal cancer was added - dose optimization. This was the direct consequence of the accurate and central position of the distal $10 \mathrm{~cm}$ of the applicator due to the inflatable balloons. The noticed consistency of the BCEA positioning resulted in the possibility to deliver non-uniform dose along the catheter, which could result in an increase of the dose to the cancerous tissue, whereas the dose to the healthy mucosa and other organs at risk was minimized. This can be achieved, irrespective of the curvature along the treatment length, since the dwell times can be adjusted to maintain the desired dose distributions. The rigorous dosimetric comparison between the BCEA and EA can be a part of future studies.

Long-term toxicities and the correlation between the formation of a fistula or ulceration and the novel design of the BCEA are the topics that can be additionally investigated using the data of more patients treated with the novel BCEA and longer follow-up. Due to the limited number of patients, it is not yet possible to conclude if the patients benefit from the treatment using the centrally placed applicator. Furthermore, due to the provision to additionally optimize the dose, there exists a possibility of dose escalations for certain patients, depending on their anatomy and the spread of disease. The initial implementation of this applicator required strict and careful testing, especially in the determination of the accurate treatment length that would allow the radioactive source to be sent to the most distal position (first dwell position). Multiple tests and an interobserver agreement are required since the inaccurate results of this test can potentially offset the whole treatment, causing adverse events. Therefore, the treatment length and BCEA applicator positioning should be evaluated before each fraction.

\section{Conclusions}

In this study, the relevant steps in commissioning, clinical implementation, treatment planning, and quality assurance for the novel BCEA were presented. The initial experience revealed that such technique was beneficial in the treatment of the curved anatomy of the lesion due to the improved repeatability and consistency of the delivered fractional dose to the patient. The inflatable balloons assisted in placing the catheter centrally and consistently with respect to the clinical target. A larger cohort of patients will be required for additional conclusions related to the treatment outcome and potential long-term clinical benefits of radiation treatment using this clinical device.

\section{Disclosure}

Authors declare no conflict of interest. 


\section{References}

1. Gaspar LE, Nag S, Herskovic A et al. Clinical Research Committee. American Brachytherapy Society (ABS) consensus guidelines for brachytherapy of esophageal cancer. Int J Radiat Oncol Biol Phys 1997; 38: 127-132.

2. Gaspar LE. Radiation therapy for esophageal cancer: improving the therapeutic ratio. Semin Radiat Oncol 1994; 4: 192-201.

3. Sharma V, Mahantshetty U, Dinshaw KA et al. Palliation of advanced/recurrent esophageal carcinoma with high-dose-rate brachytherapy. Int J Radiat Oncol Biol Phys 2002; 52: 310-315.

4. Mahantshetty U, Dinshaw K, Sharma V. Palliation of advanced/recurrent carcinoma esophagus with intraluminal brachytherapy. Ind J Thor Cardiovascr Surg 2003; 19: 119-123.

5. Skowronek J, Adamska K, Suwalska M et al. Palliative HDR brachytherapy in treatment of advanced esophageal cancer. Rep Pract Oncol Radiother 2000; 5: 111-119.

6. Wong Hee Kam S, Rivera S, Hennequin C et al. Salvage highdose-rate brachytherapy for esophageal cancer in previously irradiated patients: A retrospective analysis. Brachytherapy 2015; 14: 531-536.

7. Hishikawa Y, Kurisu K, Taniguchi M et al. High-dose-rate intraluminal brachytherapy (HDRIBT) for esophageal cancer. Int J Radiat Oncol Biol Phys 1991; 21: 1133-1135.

8. Sur RK, Singh DP, Sharma SC et al. Radiation therapy of esophageal cancer: role of high dose rate brachytherapy. Int J Radiat Oncol Biol Phys 1992; 22: 1043-1046.

9. Sur RK, Levin CV, Donde B et al. Prospective randomized trial of HDR brachytherapy as a sole modality in palliation of advanced esophageal carcinoma - an International Atomic Energy Agency study. Int J Radiat Oncol Biol Phys 2002; 53 : 127-133.

10. Yorozu A, Toya K, Dokiya T. Long-term results of concurrent chemoradiotherapy followed by high dose rate brachytherapy for T2-3N0-1M0 esophageal carcinoma. Esophagus 2006; 3: 1-5.

11. Steyerberg EW, Homs MY, Stokvis A et al. SIREC Study Group. Stent placement or brachytherapy for palliation of dysphagia from esophageal cancer: a prognostic model to guide treatment selection. Gastrointest Endosc 2005; 62: 333-340.

12. Bergquist H, Wenger U, Johnsson E et al. Stent insertion or endoluminal brachytherapy as palliation of patients with advanced cancer of the esophagus and gastroesophageal junction. Results of a randomized controlled clinical trial. Dis Esophagus 2005; 18: 131-139.

13. Liu H, Wang $Q$, Wan $X$ et al. Californium-252 neutron brachytherapy combined with external beam radiotherapy for esophageal cancer: Long-term treatment results. Brachytherapy 2014; 13: 514-521.

14. Gaspar LE, Winter K, Kocha WI et al. A phase I/II study of external beam radiation, brachytherapy, and concurrent chemotherapy for patients with localized carcinoma of the esophagus (Radiation Therapy Oncology Group Study 9207). Cancer 2000; 88: 988-995.

15. Muijs CT, Beukema JC, Mul VE et al. External beam radiotherapy combined with intraluminal brachytherapy in esophageal carcinoma. Radiother Oncol 2012; 102: 303-308.

16. Chauhan A, Kaur P, Annex E. Radical external beam radiotherapy with intraluminal high rate dose brachytherapy in patients with carcinoma esophagus. Int J Gastroenter 2009; 8: 1-7.

17. Okawa T, Dokiya T, Nishio $\mathrm{M}$ et al. Multi-institutional randomized trial of external radiotherapy with and without intraluminal brachytherapy for esophageal cancer in Japan. Int J Radiat Oncol Biol Phys 1999; 45: 623-628.

18. Gaspar LE, Qian C, Kocha WI et al. A phase III study of external beam radiation, brachytherapy and concurrent chemotherapy in localized cancer of the esophagus (RTOG 92-07):
Preliminary toxicity report. Int J Radiat Oncol Biol Phys 1997; 37: 593-599.

19. Hishikawa Y, Kurisu K, Taniguchi M et al. High-dose-rate intraluminal brachytherapy for esophageal cancer: 10 years' experience in Hyogo College of Medicine. Radiother Oncol 1991; 21: 107-114.

20. Prott FJ, Pötter R, Kovacs G et al. Magnetic resonance imaging assisted localization of tumor and applicator for brachytherapy of cancer of the esophagus (chapter). In: Tumor Response Monitoring and Treatment Planning. Springer - Verlag, Berlin-Heidelberg 1992; 607-612.

21. Rivard MJ, Coursey BM, DeWerd LA et al. Update of AAPM Task Group No. 43 Report: A revised AAPM protocol for brachytherapy dose calculations. Med Phys 2004; 31: 633-674.

22. Uniyal SC, Sharma SD, Naithani UC. Dosimetric verification of a high dose rate brachytherapy treatment planning system in homogeneous and heterogeneous media. Phys Med 2013; 29: 171-177.

23. Folkert MR, Cohen GM, Wu AJ et al. Endoluminal highdose-rate brachytherapy for early stage and recurrent esophageal cancer in medically inoperable patients. Brachytherapy 2013; 12: 463-470.

24. Bhatt L, Tirmazy S, Sothi S. Intraluminal high-dose-rate brachytherapy for palliation of dysphagia in cancer of the esophagus: initial experience at a single UK center. Dis Esophagus 2013; 26: 57-60.

25. Laskar SG, Lewis S, Agarwal JP et al. Combined brachytherapy and external beam radiation: an effective approach for palliation in esophageal cancer. J Contemp Brachytherapy 2015; 6: 453-461.

26. Lettmaier S, Strnad V. Intraluminal brachytherapy in esophageal cancer: defining its role and introducing the technique. J Contemp Brachytherapy 2014; 2: 236-241.

27. Stewart AJ, Tselis N, Albert M et al. Gastrointestinal Brachytherapy. In Brachytherapy: Applications and Techniques. Springer Publishing Company 2015; 319-354 